International Journal of Modern Physics C

Vol. 25, No. 8 (2014) 1450034 (11 pages)

(c) The Authors

DOI: 10.1142/S012918311450034X

\title{
A glimpse of fluid turbulence from the molecular scale
}

\author{
Teruhisa S. Komatsu*, Shigenori Matsumoto, \\ Takashi Shimada and Nobuyasu Ito \\ Department of Applied Physics \\ The University of Tokyo, Hongo, Bunkyo \\ Tokyo 113-8656, Japan \\ *komatsu@serow.t.u-tokyo.ac.jp
}

Received 16 October 2013

Accepted 28 January 2014

Published 3 March 2014

\begin{abstract}
Large-scale molecular dynamics (MD) simulations of freely decaying turbulence in threedimensional space are reported. Fluid components are defined from the microscopic states by eliminating thermal components from the coarse-grained fields. The energy spectrum of the fluid components is observed to scale reasonably well according to Kolmogorov scaling determined from the energy dissipation rate and the viscosity of the fluid, even though the Kolmogorov length is of the order of the molecular scale.
\end{abstract}

Keywords: Molecular dynamics; turbulence; statistical mechanics; fluid dynamics; nanofluidics.

PACS Nos.: 05.10.Ln, 47.11.Mn.

\section{Introduction}

Starting from appropriate constitutive equations of motion - not from molecular scale - often gives clear insight to the system. However, such approaches also have some drawbacks: The underlying constitutive equations might be valid only in the limited conditions, and they are often violated or undetermined in the new, intermediate or extreme conditions. Molecular descriptions are much more robust in this regard, but the problem of the enormously large-scale gap between microscopic and macroscopic (or mesoscopic) scales remains.

A calculation of molecular dynamic (MD) motion to study various equilibrium and nonequilibrium phenomena was pioneered in the mid-20th century. Encouraged by the fact that even the small 32-particle system of Alder and Wainwright ${ }^{1}$ captures the tail of the branch in larger systems, researchers have developed methods of MD simulation with the aid of the exponential growth of computational power, the 


\section{T. S. Komatsu et al.}

so-called Moore's law. ${ }^{2}$ For example, MD simulations of $10^{4}$ - to $10^{5}$-particle systems have been employed to study hydrodynamics at low Reynolds number in two dimensions $^{3-5}$ and heat conduction in two- and three-dimensional particle systems. ${ }^{6,7}$

Recent developments in parallel computers are further accelerating the speed of growth beyond Moore's law, and huge (>10 ${ }^{8}$-particle system) simulations are becoming realistic, and the realms reachable from the molecular scale are getting broader. Although the molecular scale simulations are computationally intensive, they could become reasonable, realistic approaches to some extreme classes of phenomena such as nanofluidics in high Reynolds number, where the underlying constitutive equations for a continuum description might fail. Because recent engineering applications are increasingly confronted with such extreme systems, the role of molecular scale simulations is becoming more important.

Here, we focus on molecular scale simulations of turbulent flow, which has been one of the most challenging targets in hydrodynamics. In the fluctuating turbulent fluid, energy currents sustain the hierarchical scale structures, which can usually be approached by starting from coarse-grained phenomenological descriptions and assuming clear separation between macroscopic (hydrodynamic) and microscopic (molecular) scales. However, the validity of such phenomenological approaches can be doubtful, especially when the hydrodynamic scale is comparable to the molecular scale, where fluid fluctuations compete with molecular scale fluctuations. Then, a clear starting point from the molecular scale would have merit. The aim of the present research is not to replace all fluid simulations with molecular scale simulations, but rather to test challenging simulations and check whether molecular scale simulations are feasible. Such simulations would play a complementary role under certain extreme conditions in which the continuum fluid description fails.

Resolving turbulent flow from the molecular scale requires a huge number of molecular particles, and larger systems need longer simulation time scale. The present parallel computer architecture allows us to treat larger systems, although it is not yet easy to treat longer time simulations. Thus, it would be a useful exercise to examine how well we can approach turbulence from the molecular scale by using current computer systems.

\section{Methods}

\subsection{MD simulation}

In this paper, we focus on MD simulation of freely decaying fluid flow starting from an initial velocity profile, the Taylor-Green vortex (TGV), ${ }^{8,9}$ which is one of the most well-known benchmark systems for fluid simulation. The velocity profile of the TGV,

$$
\boldsymbol{u}^{\mathrm{TG}}(x, y, z)=U_{0}\left(\begin{array}{c}
\sin (2 \pi x / L) \cos (2 \pi y / L) \cos (2 \pi z / L) \\
-\cos (2 \pi x / L) \sin (2 \pi y / L) \cos (2 \pi z / L) \\
0
\end{array}\right)
$$


is composed of twisted vortex pairs in an $L^{3}$ rectangular periodic box, and its energy per mass is $U_{0}^{2} / 8$.

In the molecular description, the fluid is composed of $N\left(\approx 10^{8}\right)$ identical particles of mass $m$. The time developments of the $i$ th particle's position $\boldsymbol{r}_{i}$ and momentum $\boldsymbol{p}_{i}$ are described as

$$
\begin{aligned}
& (d / d t) \boldsymbol{r}_{i}=\boldsymbol{p}_{i} / m, \\
& (d / d t) \boldsymbol{p}_{i}=-\sum_{j \neq i} \partial_{\boldsymbol{r}_{i}} V\left(\left|\boldsymbol{r}_{i}-\boldsymbol{r}_{j}\right|\right)+\boldsymbol{f}_{\text {wall }},
\end{aligned}
$$

with simple repulsive model for the interaction potential $V(r)$,

$$
V(r)=Y\left(r_{c}-r\right)^{a},
$$

for $r<r_{c}$ and $V(r)=0$ for $r \geq r_{c}$, where $r$ is the distance between the particles.

By utilizing the symmetry of the TGV, the present MD simulation is performed in an $(L / 2)^{3}$ rectangular box with slip boundary conditions at the surfaces of the box, not in the $L^{3}$ periodic box. This reduces the simulation costs by a factor of $\sim 8$. Although one may have concerns about the correctness of this treatment, we have confirmed that almost the same energy spectra are obtained for the periodic boundary system and the slip boundary system $(L=400)$, at least within the order of the turnover time scale.

To realize the slip boundary conditions at the surfaces of the box, each particle near the $\xi(=\{x, y, z\})$ wall is (additionally) forced by a self-mirror image with the interaction potential $V(r)$, i.e.

$$
\boldsymbol{f}_{\text {wall }}=-\partial_{\xi_{i}} V\left(2\left|\xi_{i}-\xi_{\text {wall }}\right|\right) \hat{\boldsymbol{e}}_{\xi},
$$

where $\xi_{i}\left(\xi_{\text {wall }}\right)$ is the $\xi$-component of the $i$ th particle (wall) position and $\hat{\boldsymbol{e}}_{\xi}$ is the unit vector in the $\xi$-direction.

The time developments of the MD system are calculated by using Hamiltonian dynamics (2) starting from a microscopic state of particles at $t=0$ whose macroscopic properties correspond to a velocity field $\boldsymbol{u}^{\mathrm{TG}}(x, y, z)$, a kinetic temperature $T_{0}$ (with a Boltzmann constant of unity) and a uniform number density $\bar{\rho}$. The initial microscopic states are prepared as follows. The particle positions $\left\{\boldsymbol{r}_{i}\right\}$ are located on FCC sites whose lattice constant is adjusted to reproduce the specified number density $\bar{\rho}$. The particle momentum $\boldsymbol{p}_{i}$ is taken from a Gaussian distribution with the amplitude $\sqrt{m T_{0}}$. The origin of time is defined just after the execution of short transient simulations (typically $t_{\mathrm{tr}}=10$ ) from these configurations. Then, the initial conditions for the molecular fluid are prepared by adding position-dependent velocity components $m \boldsymbol{u}^{\mathrm{TG}}\left(\boldsymbol{r}_{i}\right)$ to each particle's $\boldsymbol{p}_{i}$.

Unless otherwise specified, we have used the parameter set $m=1, r_{c}=1, a=2$ and $Y=500$. Under the conditions presented in this paper, particles with these parameters are sufficiently hard to ensure that the contribution of the potential energy to the total energy was less than a few percent. The time developments of the configuration $\left\{\boldsymbol{r}_{i}(t), \boldsymbol{p}_{i}(t)\right\}$ are calculated using a second-order symplectic integrator 


\section{T. S. Komatsu et al.}

with time-step $\Delta t=4 \times 10^{-4}$. Our simulation code was developed based on the flat MPI parallel code, MDACP ${ }^{10}$ which can treat short-ranged interacting particle systems with a high efficiency of parallelization. Main simulation runs utilized 256 nodes $\times 64$ threads on SR16000M1 (see Appendix A for detail).

\subsection{Coarse-grained field quantities}

From microscopic information such as the positions and the velocities of the particles, we calculate those local averages to obtain the coarse-grained field quantities. First, we grid the system into cubic cells so that a cell labeled $\left(n_{x}, n_{y}, n_{z}\right)$ occupies a volume $n_{\xi} l_{\text {cell }} \leq \xi<\left(n_{\xi}+1\right) l_{\text {cell }}$ (for $\xi=\{x, y, z\}$ ). Then the coarse-grained field quantities are defined at each center of the cell, $\boldsymbol{r}=\left(\left(n_{x}+1 / 2\right) l_{\text {cell }},\left(n_{y}+1 / 2\right) l_{\text {cell }}\right.$, $\left.\left(n_{z}+1 / 2\right) l_{\text {cell }}\right)$. To be specific, we define (number) density fields $\rho(\boldsymbol{r}):=$ $\sum_{i} w\left(\boldsymbol{r}, \boldsymbol{r}_{i}\right) 1 / l_{\text {cell }}^{3}$, velocity fields $\boldsymbol{u}(\boldsymbol{r}):=\sum_{i} w\left(\boldsymbol{r}, \boldsymbol{r}_{i}\right) \boldsymbol{p}_{i} / m \rho(\boldsymbol{r}) l_{\text {cell }}^{3}$ and so on, where the function $w\left(\boldsymbol{r}, \boldsymbol{r}_{i}\right)$ takes the value 1 , if the particle $i$ is in the cubic cell and otherwise 0 .

\section{Results}

Although the total energy of the MD system is conserved, coherent "fluid" motion decays in the course of time. In order to observe such a fluid property from the MD data, the fluid components must be extracted. Hence, we observe the coarse-grained field quantities defined in the previous section. Typical snapshots of the observed velocity fields in our MD simulation are shown in Fig. 1.

These fields in $(L / 2)^{3}$ space are converted to those in $L^{3}$ space before spectrum analysis. The "energy" per mass described by these coarse-grained fields is

$$
\frac{1}{m \bar{\rho} L^{3}} \int d \boldsymbol{r} \frac{m \rho(\boldsymbol{r})}{2}|\boldsymbol{u}(\boldsymbol{r})|^{2}
$$

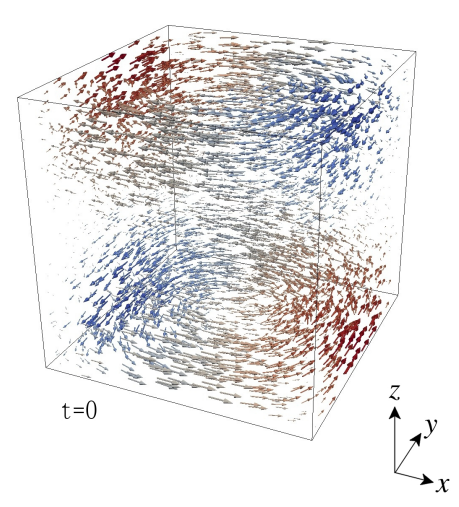

(a)

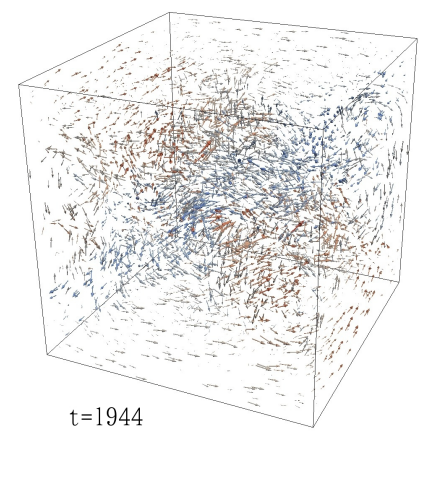

(b)

Fig. 1. (Color online) Velocity fields observed at (a) $t=0$ and (b) $t=1944$ in the MD simulation box of $(L / 2)^{3}\left(L / 2=1080, N=3.779136 \times 10^{8}, U_{0}=2, T_{0}=0.33, l_{\text {cell }}=10\right)$. Color encodes $y$ components of the velocities. 


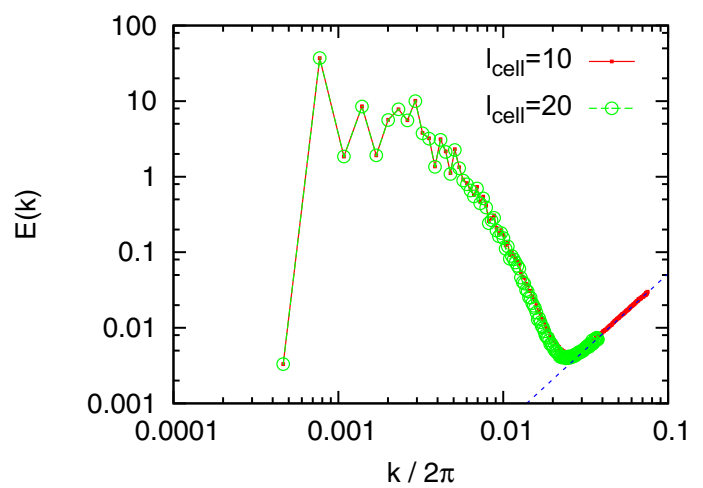

Fig. 2. (Color online) Energy spectrum for $L / 2=1080, T_{0}=0.33, \bar{\rho}=0.30, U_{0}=2.0$ and $t=2160$. Two cases of cell size $l_{\text {cell }}=10$ (small red squares) and 20 (green circles) are shown. The broken line is $(3 T / m$ $\bar{\rho})(k / 2 \pi)^{2}$ with $T=0.52$, which is the temperature obtained from the averaged variance of the molecular velocities in each cell $\left(l_{\text {cell }}=10\right)$.

which can be written as the $k$-shell averaged power spectrum

$$
\frac{1}{2 L^{3}} \int d \boldsymbol{r}|\boldsymbol{v}(\boldsymbol{r})|^{2}=\frac{1}{2} \sum_{k}|\tilde{\boldsymbol{v}}(\boldsymbol{k})|^{2}=: \int d k E(k),
$$

of the vector field

$$
\boldsymbol{v}(\boldsymbol{r}):=\boldsymbol{u}(\boldsymbol{r}) \sqrt{\rho(\boldsymbol{r}) / \bar{\rho}}=: \sum_{\boldsymbol{k}} \tilde{\boldsymbol{v}}(\boldsymbol{k}) \mathrm{e}^{i \boldsymbol{k} \cdot \boldsymbol{r}}
$$

In the energy spectrum $E(k)$ as shown in Fig. 2, thermally equilibrated (equipartitioned) spectrum proportional to $k^{2}$ is observed in the small-scale (highwave-number) region after some transient time. When $l_{\text {cell }}$ is varied, only the higher-wave-number cutoff of the spectrum is varied, while lower-wave-number spectrum is unchanged. This suggests that the $k^{2}$ branch corresponds to small-scale random thermal modes. In the following, we take $l_{\text {cell }}=10$ to observe the entire scale range from microscopic to hydrodynamic in the energy spectrum.

When the equipartition with temperature $T$ is satisfied, $\tilde{\boldsymbol{v}}(\boldsymbol{k})$ becomes $\boldsymbol{k}$-independent and the kinetic energy for each cell (of mass $m \bar{\rho} l_{\text {cell }}^{3}$ ) is equal to $(3 / 2) T$, i.e.

$$
\left(m \bar{\rho} l_{\text {cell }}^{3} / 2\right)\left(L / l_{\text {cell }}\right)^{3}|\tilde{\boldsymbol{v}}(\boldsymbol{k})|^{2}=(3 / 2) T,
$$

where we note that $\sum_{k} 1=\left(L / l_{\text {cell }}\right){ }^{3}$ Then by multiplying the density of states in the $k$-shell, the energy spectrum in thermal equilibrium is expressed as

$$
E_{\text {eq }}(k)=\left(4 \pi k^{2} / k_{\min }^{3}\right)\left(3 T / 2 m \bar{\rho} L^{3}\right)=(3 T / m \bar{\rho})(k / 2 \pi)^{2},
$$

where $k_{\min }:=2 \pi / L$. The values of temperature $T$ estimated from the variance of molecular velocities in each cell are consistent with those estimated from $E(k)$ (see Fig. 2). The time developments of $T$ are shown in Fig. 3, where the molecular fluid seems to be heated during the course of relaxation. 


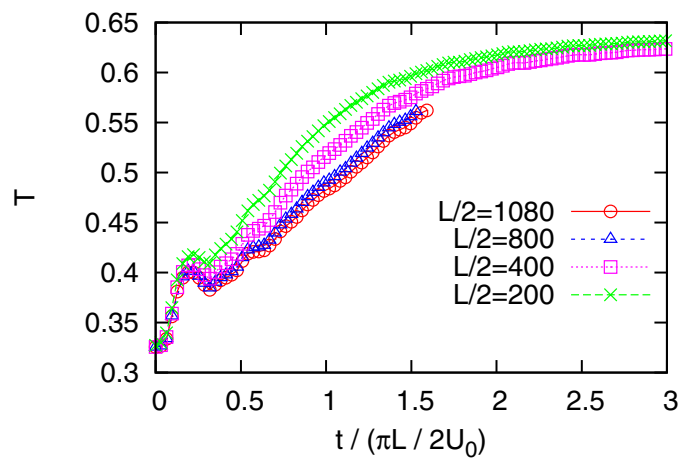

Fig. 3. (Color online) Time developments of temperature $T$ determined from the averaged variance of the molecular velocities in each cell. $T_{0}=0.33, \bar{\rho}=0.30$ and $U_{0}=2.0$. (Note that this definition of temperature might not be reliable during the early stage.) At the final equilibrium state $(t \rightarrow \infty)$, the temperature would become $T_{\infty}=T_{0}+(2 / 3)(m / 8) U_{0}^{2}=0.66$.

Eliminating these thermally equilibrated components, we estimate the fluid components from the MD data. For example, the energy of the fluid components, $E^{\text {fluid }}$, is extracted from the fitting of the function $\int_{0}^{k} d k^{\prime} E\left(k^{\prime}\right)$ to the form $a k^{3}+E^{\text {fluid }}$ in the high-wave-number region.

We also observe the enstrophy, which is a measure of the vorticity field $\operatorname{rot} \boldsymbol{u}(\boldsymbol{r})$. The enstrophy spectrum is defined by

$$
\int d k \Omega(k):=\int d \boldsymbol{r}|\operatorname{rot} \boldsymbol{u}(\boldsymbol{r})|^{2} /\left(2 L^{3}\right) .
$$

Similarly to the energy, the enstrophy of the fluid components, $\Omega^{\text {fluid }}$, is extracted from the fitting to $a^{\prime} k^{5}+\Omega^{\text {fluid }}$, where the terms $\operatorname{rot} \boldsymbol{u}(\boldsymbol{r})$ can be calculated in Fourier space.

The time developments of $E^{\text {fluid }}$ for various system sizes $L$ are shown in Fig. 4(a). One can observe that decay of the fluid energy roughly corresponds to the increase in the temperature in Fig. 3.

In Fig. 4(b), the divergence-free (DF) components of energy are also shown based on the vector decomposition

$$
\tilde{\boldsymbol{v}}_{\mathrm{RF}}(\boldsymbol{k}):=\boldsymbol{k}[\boldsymbol{k} \cdot \tilde{\boldsymbol{v}}(\boldsymbol{k})] / k^{2}, \quad \tilde{\boldsymbol{v}}_{\mathrm{DF}}(\boldsymbol{k}):=\tilde{\boldsymbol{v}}(\boldsymbol{k})-\tilde{\boldsymbol{v}}_{\mathrm{RF}}(\boldsymbol{k}),
$$

in which $\tilde{\boldsymbol{v}}_{\mathrm{RF}}(\boldsymbol{k})$ are the rotational-free $(\mathrm{RF})$ components and $|\tilde{\boldsymbol{v}}(\boldsymbol{k})|^{2}=\left|\tilde{\boldsymbol{v}}_{\mathrm{RF}}(\boldsymbol{k})\right|^{2}+$ $\left|\tilde{\boldsymbol{v}}_{\mathrm{DF}}(\boldsymbol{k})\right|^{2}$.

Comparing $E^{\text {fluid }}$ and the energy of the DF components $\left(E_{\mathrm{DF}}^{\text {fluid }}\right)$ in Fig. 4(b), one notes that the major contribution to the energy comes from the DF components. The bumpy shape during the early stage is mainly due to the RF components (at least for this case) and comes from sound wave propagation in the system. Since the present $U_{0}$ is comparable to the speed of sound, the density profile becomes rather inhomogeneous during the early stage of the simulation, but during the later stage, it 


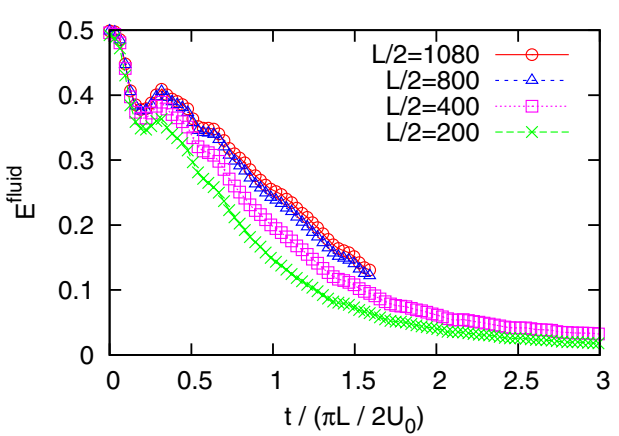

(a)

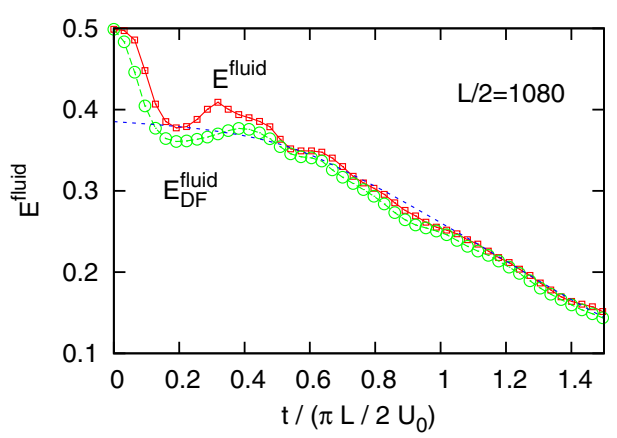

(b)

Fig. 4. (Color online) (a) Time developments of $E^{\text {fluid }}$ for $\bar{\rho}=0.30, T_{0}=0.33$ and $U_{0}=2.0$. (b) Comparison between $E^{\text {fluid }}$ (squares) and $E_{\mathrm{DF}}^{\text {fluid }}$ (circles). The two curves coincides at later stages. The broken line is the estimation of fluid energy by $E^{\text {fluid }}\left(t_{*}\right)+2 \nu \int_{t}^{t_{*}} d t^{\prime} \Omega^{\text {fluid }}\left(t^{\prime}\right)$ with $\nu=0.72$ fitted during the later stage.

relaxes toward the homogeneous one. Thus, approximating the fluid as incompressible might be allowed during the later stage.

Since $E^{\text {fluid }}$ has been determined, the energy dissipation rate $\epsilon$ can be estimated. In case of an incompressible Navier-Stokes fluid, $\epsilon$ and $\Omega^{\text {fluid }}$ are related by

$$
\epsilon=2 \nu \Omega^{\text {fluid }},
$$

where $\nu$ is the dynamic viscosity. By assuming this relation holds during the later stage for the present system, the values of $\nu$ can be fitted from the data. These values of $\nu$ are consistent with those of $\nu_{P}$ obtained in the other series of simulations for Poiseuille flow (see Fig. 5).

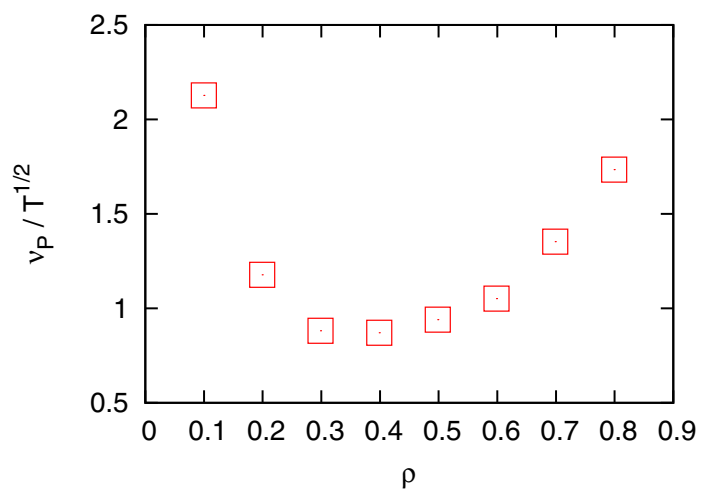

Fig. 5. (Color online) Dynamic viscosity $\nu_{P}$ obtained from Poiseuille flow simulated in a periodic box $\left(L_{w} \times 100 \times 100\right)$, where fixed wall conditions are imposed by a Langevin thermostat in the flat plate region $(8 \times 100 \times 100)$. The velocity profile (in the central region excluding the vicinity of the wall) is fitted to the parabolic form $u_{z}(x)=\left(g / 2 \nu_{P}\right) x\left(L_{w}-x\right)$, where $g$ is the gravitational acceleration and $L_{w}$ is the wall-to-wall length $\left(g=0.001, L_{w}=50\right.$ and $\left.T=0.5\right)$. Because the interaction potential is hard for the given temperature $T$, the dynamic viscosity $\nu_{P}$ would be scaled as $\sqrt{T}$. 


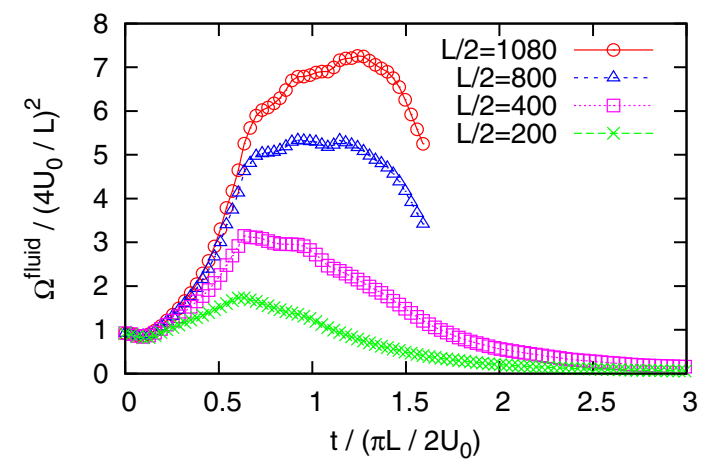

Fig. 6. (Color online) Time developments of $\Omega^{\text {fluid }}$ for $\bar{\rho}=0.30, T_{0}=0.33$ and $U_{0}=2.0$.

As shown in Fig. $6, \Omega^{\text {fluid }}$ grows in time and takes a maximum value at time $t_{*}$ (comparable to the order of the turnover time $\left.\pi L / 2 U_{0}\right)$. Around $t=t_{*}$, the production of small-scale structure in the fluid mode and the relaxation toward equilibrium balance in some sense. The maximum values of enstrophy normalized by its initial one increase with $L$, which suggests that larger systems can produce wider scale structures.

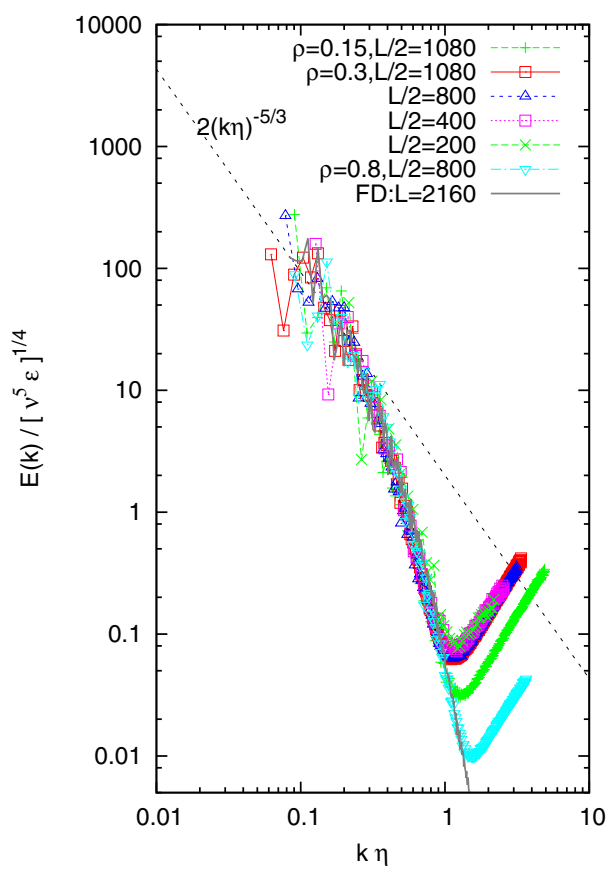

Fig. 7. (Color online) Scaled energy spectrum $E(k) /\left(\nu^{5} \epsilon\right)^{1 / 4}$. The parameters are shown in Table 1. The dotted line $2(k \eta)^{-5 / 3}$ is drawn to guide to eye. The FD result is obtained from the simulation of the incompressible Navier-Stokes equation $\left(L=2160, \nu=0.7\right.$ and $\left.U_{0}=1.7\right)$ by using a pseudo-spectrum method $\left(200^{3}\right.$ modes $)$ with a simple $3 / 2$ padding aliasing free method. ${ }^{11}$ 
Table 1. Parameters and observables at $t=\left(\pi L / 2 U_{0}\right) \tilde{t}_{*}$. Listed are fluid energy $E$ and enstrophy $\Omega$ determined from the spectra (see $E^{\text {fluid }}$ and $\Omega^{\text {fluid }}$ in the text), energy dissipation rate $\epsilon$ from the decay of $E$, temperature $T$ from the variance of velocities in each cell, dynamic viscosity $\nu:=\epsilon / 2 \Omega$, Kolmogorov length $\eta:=\left(\nu^{3} / \epsilon\right)^{1 / 4}$ and Reynolds number $R:=(L / 2) U / \nu$ with characteristic velocity $U:=(2 E / 3)^{1 / 2}$. For all MD simulations in this table, $U_{0}=2$ and $T_{0}=0.33$.

\begin{tabular}{lccccrcrrr}
\hline$L / 2$ & $\bar{\rho}$ & $\tilde{t}_{*}$ & $E$ & $10^{4} \Omega$ & $10^{4} \epsilon$ & $T$ & $\nu$ & $\eta$ & $R$ \\
\hline 1080 & 0.15 & 0.83 & 0.25 & 0.51 & 1.09 & 0.490 & 1.08 & 10.4 & 410 \\
1080 & 0.30 & 1.24 & 0.20 & 1.00 & 1.44 & 0.515 & 0.72 & 7.1 & 548 \\
800 & 0.30 & 0.92 & 0.26 & 1.33 & 1.91 & 0.480 & 0.72 & 6.6 & 464 \\
400 & 0.30 & 0.64 & 0.31 & 3.15 & 4.61 & 0.447 & 0.73 & 5.4 & 249 \\
200 & 0.30 & 0.64 & 0.26 & 6.93 & 10.84 & 0.477 & 0.78 & 4.6 & 106 \\
800 & 0.80 & 0.95 & 0.25 & 1.53 & 3.18 & 0.435 & 1.04 & 7.7 & 314 \\
\hline
\end{tabular}

Now let us study the energy spectrum at $t=t_{*}$, the time at which the flow is expected to be most turbulent. Figure 7 shows the energy spectra for various $L$ values listed in Table 1 (where, for clarity, only the higher-wave-number range is shown), in which the spectrum obtained from continuum fluid dynamics (FD) simulation is also shown. First, one notes that the spectra scale reasonably well-(including the FD results) according to the Kolmogorov scaling is determined by $\epsilon$ and $\nu{ }^{12-14}$ Second, in the present case, crossover scales to thermal modes are rather close to the Kolmogorov length

$$
\eta:=\left(\nu^{3} / \epsilon\right)^{1 / 4}
$$

and clear deviation of the (dissipation) spectrum is not noticed. Although the system size of the present simulation is still limited, $E(k)$ seems to approach Kolmogorov spectrum $(k \eta)^{-5 / 3}$ around $k \eta \leq 0.2$. Additional large-scale simulations would demonstrate the spectrum more clearly.

\section{Discussion}

In this paper, we have reported MD simulations of TGV to explore fluid turbulence from a simple molecular starting point. We have determined the energy and enstrophy of the fluid mode from the cell-averaged MD data by eliminating thermal modes associated with microscopic molecular motion. The extracted observables of the fluid mode during the later stage imply that the fluid can be treated as incompressible. The obtained energy spectrum scales well according to Kolmogorov scaling, even though the spectrum around the Kolmogorov length $(k \eta \sim 2 \pi)$ is dominated by the thermal modes. Below around $k \eta \leq 0.2$, the spectrum seems to approach a power law, which might indicate a glimpse of turbulence (or that we have reached the smallest unit of turbulence) from the molecular scale.

For efficient usage of computational power to resolve turbulent flow, a rather strong (supersonic) velocity field is initially imposed; this contributes to narrowing the separation between molecular and fluid scales. The resulting Kolmogorov length 
is of the order of several particle diameters, which could be of the order of the microscopic scale, the mean free path of the particles. As seen in Table 1, $\eta$ increase with $L$ as $\eta \propto L^{1 / 4}$. This relationship holds because the dissipation rate is mainly determined by the macroscopic time scale $L / U_{0}$, and thus roughly $\epsilon \propto L^{-1}$.

Usually, the Kolmogorov length is sufficiently large compared to the microscopic scale, as one can simply assume the separation of scales between fluid and microscopic ones. For example, from a rough estimation for atmospheric gas $\left(\nu \sim 10^{-2} \mathrm{~m}^{2} / \mathrm{s}\right.$ and $m \bar{\rho} \sim 1 \mathrm{~kg} / \mathrm{m}^{3}$ ) under conditions such that the large-scale velocity, large-scale length and energy dissipation rate are $U \sim 10 \mathrm{~m} / \mathrm{s}, L \sim 10 \mathrm{~m}$ and $\epsilon \sim U^{3} / L$ (solely determined from the large-scale motion), respectively, the resulting Kolmogorov length is $\eta \sim 10^{-2} \mathrm{~m}$, which is $10^{5}$ times the order of the mean free path. Correspondingly, the height of the scaled energy spectrum for the thermal branch at $k \eta=1$ is estimated to be $<10^{-15}$ from Eq. (9) divided by $\left(\nu^{5} \epsilon\right)^{1 / 4}$. Thus, it is rather hard to observe the thermal branch at the tail of the dissipation spectrum under usual conditions.

However, in this study, the Kolmogorov length becomes comparable to the microscopic scale, i.e. turbulent fluctuations compete with thermal fluctuations, but the energy spectrum still seems to be merely a superposition of or crossover between fluid and thermal modes. This observation is suggestive for nanofluidics in extreme conditions. Although apparent interference between molecular and fluid scales has not been noticed in the present observation, further detailed studies may yield some insight. An ingenious setup to precisely observe microscopic states in macroscopically steady turbulent states will be essential to proceed.

It is a benefit of MD simulation that we can observe turbulence directly from the molecular scale. Observations from the molecular scale up to and beyond the Kolmogorov length will supplement observations based on continuum descriptions and be expected to become a complementary method, especially for systems under special or extreme conditions.

In addition to those academic interests, direct simulation from the molecular scale will have merits in some engineering situations, for example, the flow of complex fluids that contains phase transitions, impact ruptures or coalescence. For such complex flows, ambiguities may remain in the constitutive equations and so clear starting points from the molecular scale will attract much attention.

\section{Acknowledgments}

We wish to thank M. Miyama and Y. Murase for helpful advices. Numerical simulations were partly carried out by the use of the Plasma Simulator at the National Institute for Fusion Science and supported by the NIFS Collaboration Research programs (NIFS10KNSS014). This work was partly supported by Award No. KUK-I1-005-04 made by King Abdullah University of Science and Technology (KAUST). 


\section{Appendix A. Costs of Simulation}

The simulation time for $L / 2=1080$ system upto $t=2700$ using 256 nodes $\times 64$ threads on SR16000M1(POWER7) was $12 \mathrm{~h}$. Simulation at early stage was inefficient because of unbalanced loads on each thread due to the density inhomogeneity. File I/O cost is rather expensive, e.g. it took $900 \mathrm{sec}$ ( $55 \mathrm{msec}$ per thread) to write down single snapshot of particle configuration (17 GB binary file), and it seems to grow with the system size. Thus, efficient algorithm and hardware of file I/O (if particle-resolution is necessary) should be implemented for the simulation using huge number of threads.

When the system size $L$ is increased, number of particles increases with $L^{3}$ and the characteristic time scale roughly increases with $L$ for constant $U_{0}$. The former $O\left(L^{3}\right)$ can be tackled by using huge scale multi-core system. The latter $O(L)$ has direct influence on the turn-around time of single simulation.

\section{References}

1. B. J. Alder and T. E. Wainwright, J. Chem. Phys. 27, 1208 (1957).

2. G. E. Moore, Electronics 38, 114 (1965).

3. D. C. Rapaport and E. Clementi, Phys. Rev. Lett. 57, 695 (1986).

4. D. C. Rapaport, Phys. Rev. A 36, 3288 (1987).

5. T. Ishiwata, T. Murakami, S. Yukawa and N. Ito, Int. J. Mod. Phys. C 15, 1413 (2004).

6. T. Shimada, T. Murakami, S. Yukawa, K. Saito and N. Ito, J. Phys. Soc. Jpn. 69, 3150 (2000).

7. T. Murakami, T. Shimada, S. Yukawa and N. Ito, J. Phys. Soc. Jpn. 72, 1049 (2003).

8. G. I. Taylor and A. E. Green, Proc. R. Soc. Lond. A 158, 499 (1937).

9. M. Brachet, D. I. Meiron, S. A. Orszag, B. G. Nickel, R. H. Morf and U. Frisch, J. Fluid Mech. 130, 411 (1983).

10. H. Watanabe, M. Suzuki and N. Ito, Prog. Theor. Phys. 126, 203 (2011).

11. C. Canuto, M. Y. Hussaini, A. Quarteroni and T. A. Zang, Spectral Methods in Fluid Dynamics (Springer-Verlag, New York, 1988), p. 84.

12. A. N. Kolmogorov, C. R. Acad. Sci. URSS 30, 301 (1941).

13. G. K. Batchelor, Math. Proc. Cambridge Philos. Soc. 43, 533 (1947).

14. S. G. Saddoughi and S. V. Veeravalli, J. Fluid Mech. 268, 333 (1994). 